\title{
Selbstbestimmt leben - so lange wie möglich
}

\section{Ambulant betreute Demenz-Wohngemeinschaften: Stand und Entwicklungsperspektiven aus ökonomischer Sicht}

\author{
SVEN LUEKE, SARAH BLAUT, \\ GRIT ASSMANN, WOLFGANG \\ HOFFMANN, STEFFEN FLESSA \\ Sven Lueke hat an der Ernst-Moritz-Arndt- \\ Universität Greifswald im Lehrstuhl für \\ Allgemeine Betriebswirtschaftslehre \\ und Gesundheitsmanagement sowie am \\ Deutschen Zentrum für Neurodegenera- \\ tive Erkrankungen am Standort Rostock/ \\ Greifswald als Wissenschaftlicher Mit- \\ arbeiter gearbeitet. Schwerpunktmäßig \\ beschäftigte er sich dort mit Wirtschaft- \\ lichkeitsanalysen innovativer Versorgungs- \\ konzepte und Versorgungstechnologien. \\ Gegenwärtig gründet er ein Unternehmen \\ im Bereich Telemedien und ist weiterhin \\ als freiberuflicher Dozent tätig.
}

Sarah Blaut arbeitet am Lehrstuhl für Allgemeine Betriebswirtschaftslehre und Gesundheitsmanagement der Ernst-Moritz-Arndt-Universität Greifswald.

Grit Aßmann arbeitet am Deutschen Zentrum für Neurodegenerative Erkrankungen e. V. am Standort Rostock/Greifswald.

Wolfgang Hoffmann arbeitet am Deutschen Zentrum für Neurodegenerative Erkrankungen e. V. am Standort Rostock/ Greifswald und am Institut für Community Medicine, Abteilung Versorgungsepidemiologie und Community Health der Ernst-Moritz-Arndt-Universität Greifswald.

Prof. Dr. Steffen Fleßa hat den Lehrstuhl für Allgemeine Betriebswirtschaftslehre und Gesundheitsmanagement an der Ernst-Moritz-Arndt-Universität Greifswald inne. Seine Forschungsschwerpunkte sind ökonomische Evaluation von Gesundheitsdienstleistungen,

Non-Profit-Organisationen und Internationales Gesundheitswesen. www.rsf.uni-greifswald.de/flessa.html

\author{
Viele Menschen mit Demenz und ihre Familien sehen \\ in einer ambulant betreuten Wohngemeinschaft \\ eine geeignete Alternative zum Pflegeheim. Eine \\ neue Studie untersuchte die betriebswirtschaftlichen \\ Aspekte dieser Wohn- und Betreuungsform. \\ Fazit: Noch sind Pflegeheime preisgünstiger, wenn man \\ alle Kosten berücksichtig, aber die Wohngemeinschaften \\ verfügen über erhebliches Effizienzpotenzial.
}

Ambulant betreute Demenz-Wohngemeinschaften stellen eine innovative Wohn- und Versorgungsform für Menschen mit Demenz dar. Als möglicher Gegenentwurf zur stationären Versorgung in Altenpflegeheimen heben sie sich durch eine familienähnliche Atmosphäre, häusliche Strukturen und demenzspezifische Pflege- und Betreuungskonzepte ab. (1)

Die Gesundheitspolitik hat diese Wohn- und Versorgungsform als Thema aufgegriffen und mit der Verabschiedung des Pflege-NeuausrichtungsGesetzes (2) ab 1. Januar 2013 einige Verbesserungen in diesem Feld erreicht. So ist eine zusätzliche finanzielle Förderung von Demenz-Wohngemeinschaften in Höhe von 200 Euro pro Bewohner und eine Anschubfinanzierung von Neugründungen (maximal 10.000 Euro pro Wohngemeinschaft) vorgesehen.

Die zentrale Frage ist, welchen Stellenwert Demenz-Wohngemeinschaften derzeit in der Demenz-Versorgung haben (Stand) und zukünftig haben könnten (Entwicklungsperspektive), insbesondere hinsichtlich ökonomischer Rahmenbedingungen. Diese Frage soll im Folgenden auf Basis der bestehenden Literatur sowie einer Erhebung des IstZustandes beantwortet werden.

\section{Ausgangslage}

Zwei bedeutende Forschungsprojekte haben bereits einige wichtige Erkenntnisse zu den Strukturmerkmalen von betreuten Wohngemeinschaften in Deutschland geliefert:

- In einer Studie von Kremer-Preiß/Narten wurden 2003 deutschlandweit 143 betreute Wohngruppen schriftlich zu Strukturdaten befragt, wozu etwa die Wohnraumausstattung, die Charakteristika der BewohnerInnen (Alter, Pflege- und Hilfsbedarf) sowie Personal- und Kostenstruktur zählen. (3)

- Wolf-Ostermann hat 2006 eine ähnliche Untersuchung in Berlin durchgeführt, wobei u. a. ein detaillierteres Bild von den Bewohnereigenschaften aufgezeigt wurde (z. B. in Bezug auf Komorbiditäten), jedoch Kosten- und Finanzierungsaspekte vernachlässigt wurden. (4)

Beide Studien hatten zwar nicht speziell Demenz-Wohngemeinschaften in den Fokus gesetzt, konnten jedoch bei einem Großteil der Bewohnerinnen und Bewohner das Vorliegen einer Demenzerkrankung oder eine Ausrichtung der Einrichtungskonzepte auf diese spezielle Zielgruppe feststellen. 


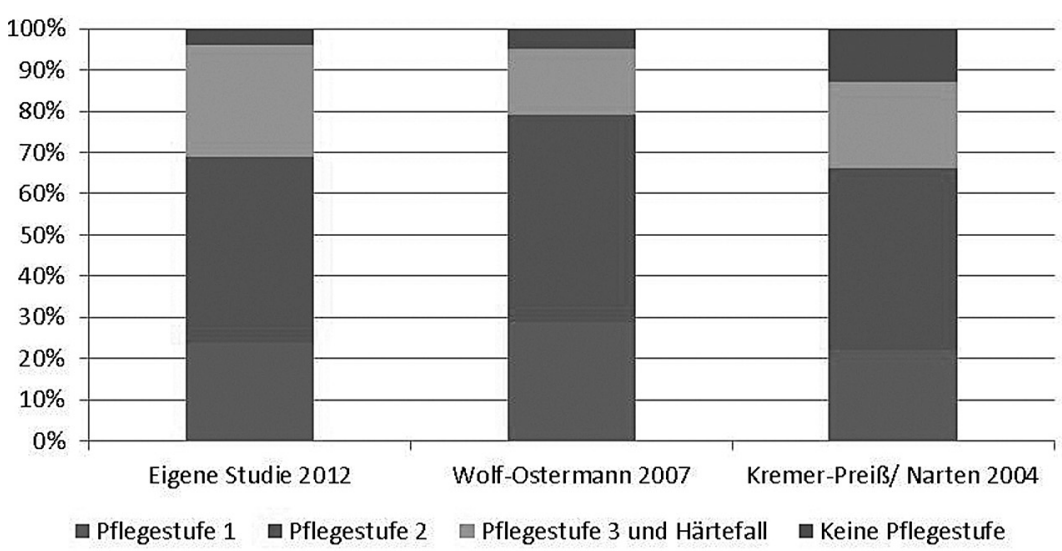

Abb. 1: Die Pflegestufe 2 stellt bei Demenz-Wohngemeinschaften die häufigste Einstufung dar, während Bewohnerinnen und Bewohner ohne Pflegestufe die Ausnahme sind.

Quelle: Eigene Darstellung

Mit dem Ziel, die Ergebnisse beider Studien zu überprüfen und mögliche Weiterentwicklungen in diesem Feld nachzuvollziehen, wurde 2012 eine eigene Studie als Kooperationsprojekt des Lehrstuhls für Allgemeine Betriebswirtschaftslehre und Gesundheitsmanagement (Universität Greifswald) und des Deutschen Zentrums für Neurodegenerative Erkrankungen (Standort: Rostock/Greifswald) durchgeführt.

Durch Internet- und Literaturrecherchen konnten 332 ambulant betreute Demenz-Wohngemeinschaften deutschlandweit identifiziert werden. Diese Einrichtungen wurden schriftlich befragt, wobei etwa 27 Prozent ( $\mathrm{N}=89$ ) der Wohngemeinschaften antworteten. Die Befragung bezog sich im Wesentlichen auf die allgemeinen Strukturmerkmale (Trägerschaft, Bewohnermerkmale, Pflegekonzepte) und im Besonderen auf die Kostenstruktur.

\section{Strukturmerkmale}

Ambulante betreute Demenz-Wohngemeinschaften sind ungleichmäßig im Bundesgebiet verteilt. NordrheinWestfalen, Niedersachsen und Berlin stellen in Deutschland etwa 65 Prozent aller Wohngemeinschaften. Ungefähr 53 Prozent der Wohngemeinschaften befinden sich in privater, 47 Prozent in freigemeinnütziger und keine einzige in öffentlicher Trägerschaft. Die häufigste Bewohnerzahl liegt zwischen sieben und neun Bewohnerinnen und Bewohner. Kremer-Preiß/Narten und Wolf-
Ostermann stellten hier als häufigste Spanne etwa sechs bis acht Bewohnerinnen und Bewohner fest. (5) Alle Studien konstatieren einen etwa 80 -prozentigen Frauenanteil. (6)

Die Pflegestufe 2 (Schwerpflegebedürftige) stellt die häufigste Einstufung der Pflegebedürftigkeit dar, während Bewohnerinnen und Bewohner ohne Pflegestufe die Ausnahme sein dürften. Zwischen 16 und 27 Prozent der Bewohnerinnen und Bewohner sind als schwerstpflegebedürftig (Pflegestufe 3) eingestuft (vgl. Abb. 1).

Auf die Frage, wie viele Bewohnerinnen und Bewohner im Laufe ihres
Lebens in einer Demenz-Wohngemeinschaft in ein Altenpflegeheim ziehen müssen, antworteten etwa 86 Prozent der Einrichtungen, dass ihr Wohnkonzept bis zum Lebensende ausgelegt sei; der restliche Anteil konnte keine genauen Angaben machen. Wolf-Ostermann konnte in ihrer Studie anhand des Stichjahres 2005 ermitteln, dass circa 71 Prozent der Bewohnerinnen und Bewohner einer Wohngemeinschaft bis zum Lebensende dort verbleiben und nur ein geringer Anteil zurück in ein häusliches Umfeld, in das Betreute Wohnen (8\%) oder ein Altenpflegeheim (9\%) zieht. (7) Die Autorin konnte auch ermitteln, dass etwa 5 Prozent der Einzüge in DemenzWohngemeinschaften aus Altenpflegeheimen kommen. (8)

\section{Kostenstruktur}

Die Kosten einer Demenz-Wohngemeinschaft setzen sich aus den Kosten für die Unterkunft (Kaltmiete plus Mietnebenkosten), für die Verpflegung (Haushaltsgeld) sowie für Pflege (SGB XI) und Betreuung zusammen.

Bei der prozentualen Gewichtung der durchschnittlichen Kosten ist zu beachten, dass es erhebliche regional- und einrichtungsspezifische Abweichungen von diesem bundesdeutschen Durchschnittswert gibt (vgl. Abb. 2).

Während die Pflegekasse durchschnittlich 2.012 Euro pro Monat für

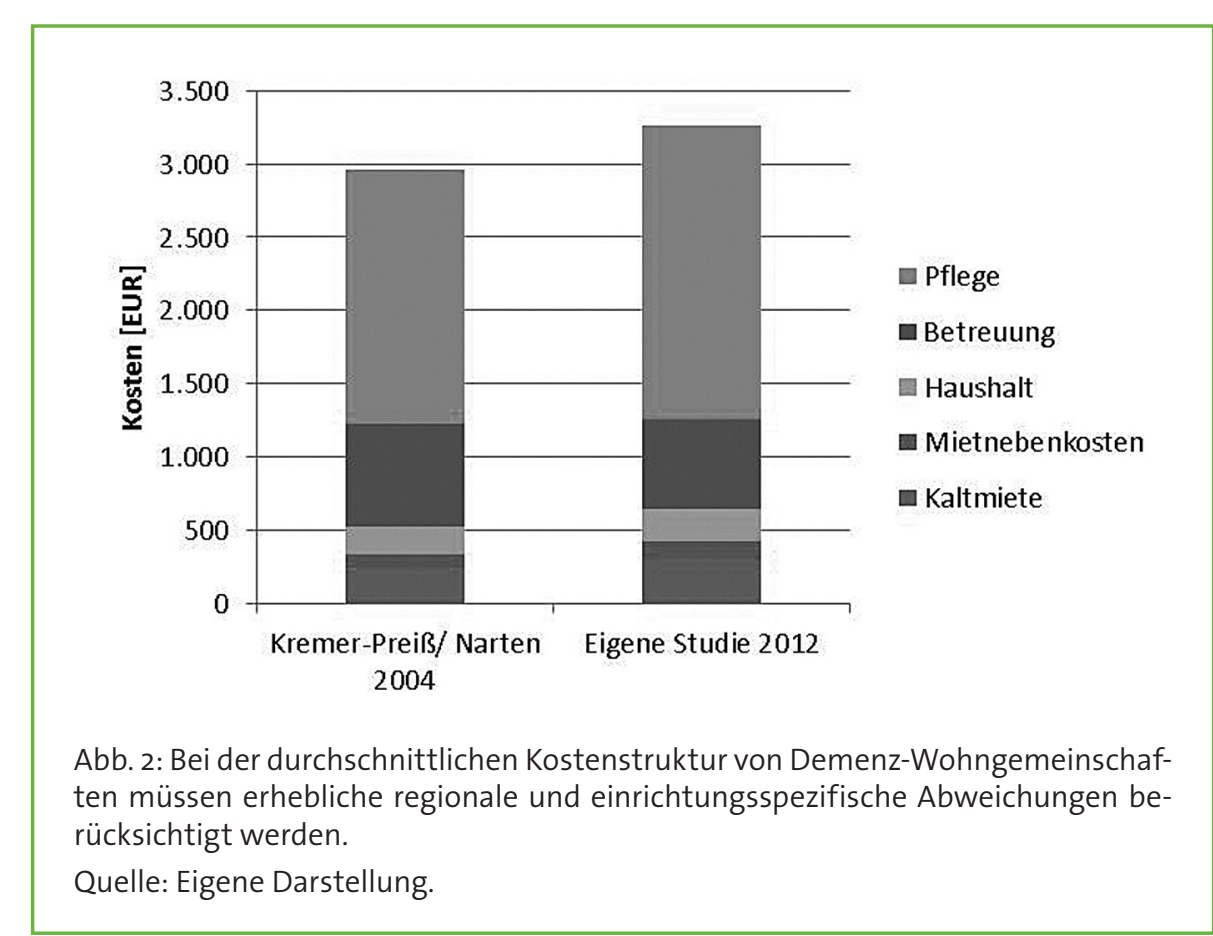


die Pflegeleistungen vollständig oder teilweise übernimmt, müssen die Bewohnerinnen und Bewohner oder die Angehörigen in der Regel für Unterkunft (518 Euro) und Verpflegung (218 Euro) sowie Betreuungsleistungen (608 Euro) aufkommen. Bei jedem zweiten Bewohner werden einzelne Kosten ganz oder teilweise durch Sozialhilfeträger übernommen. Dieser Befund wird auch von Kremer-Preiß/Narten ermittelt. (9)

\section{Entwicklungsperspektiven}

Eine Betrachtung der Strukturmerkmale ambulant betreuter DemenzWohngemeinschaften legt den Schluss nahe, dass diese nicht nur die stationäre Versorgungslandschaft ergänzen, sondern durchaus auch ersetzen können. Hierfür sprechen Einzüge aus Altenpflegeheimen in und seltene Auszüge aus Wohngemeinschaften sowie die Tatsache, dass praktisch alle Grade der Pflegebedürftigkeit versorgt werden können. Diese Substitution dürfte aller-

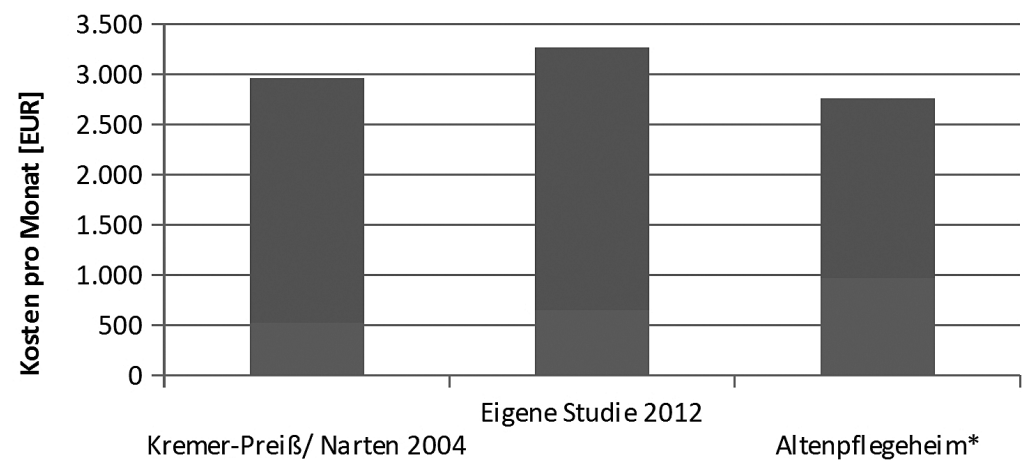

Unterkunft und Verpflegung Betreuung und Pflege

Abb. 3: Die monatlichen Gesamtkosten von Altenpflegeheimen liegen unter denjenigen von Demenz-Wohngemeinschaften.

samtkosten von Altenpflegeheimen liegen durchschnittlich 195 bis 502 Euro unter denjenigen von Demenz-Wohngemeinschaften. Zwar sind die Kosten für Unterkunft und Verpflegung in

\section{"Die meisten Demenz-}

\section{Wohngemeinschaften haben zwischen sieben und neun Bewohner»}

dings begrenzt sein, weil aus individuellen Gründen ein Leben innerhalb einer familienähnlichen Wohngemeinschaft nicht möglich sein kann.

Die Frage lautet somit, ob DemenzWohngemeinschaften auch aus ökonomischer Sicht eine Alternative zur Altenheimversorgung darstellen können (vgl. Abb. 3). Die monatlichen Ge-
Altenpflegeheimen durch die Instandhaltungsbeiträge vergleichsweise höher (um ca. 325 bis 447 Euro pro Monat), jedoch sind die Kosten für Pflege und Betreuung deutlich geringer (ca. 642 bis 827 Euro)

Aus Sicht der Pflegeversicherung ist der Einzug eines Demenzpatienten in eine Demenz-Wohngemeinschaft an- statt in ein Altenpflegeheim vorteilhaft. Für die pflegerischen Leistungen werden die ambulanten Leistungsbeiträge (Pflegesachleistung) angewendet, was gegenüber den stationären Leistungsbeiträgen bei Altenpflegeheimen eine Verringerung um 573 Euro (Pflegestufe 1) oder 179 Euro (Pflegestufe 2) pro Monat entspricht. (11) Durch die neu beschlossene finanzielle Förderung für die Betreuung in Demenz-Wohngemeinschaften in Höhe von 200 Euro relativiert sich diese Differenz.

Aus Sicht der Patienten (bzw. Angehörigen oder Sozialhilfeträgern), welche die relativ höheren Betreuungs- und teilweise Pflegekosten tragen müssen, stellt eine Demenz-Wohngemeinschaft (noch) die kostenintensivere Wohnvariante

Fortsetzung des Beitrags nach dem Jahresinhaltsverzeichnis

\section{Leben in Gemeinschaft: die ambulante Wohngemeinschaft}

Für pflegebedürftige Menschen, die Gemeinschaft suchen und in einer möglichst normalen Wohnumgebung außerhalb eines Pflegeheims leben möchten, sind ambulante Wohngemeinschaften eine gute Alternative. Im Unterschied zur stationären Wohngemeinschaft befinden sich ambulante Wohngemeinschaften in normalen Wohnhäusern in natürlicher Nachbarschaft mit nicht-pflegebedürftigen Menschen.

Hier leben Menschen in einer überschaubaren Gruppe von Bewohnern in einer sehr familiären Atmosphäre. Das persönliche
Schlafzimmer kann individuell einrichtet werden. Auch der gemeinschaftliche, großzügige Wohnbereich kann mit eigenen Gegenständen und Möbeln gestaltet werden. Unterstützt von Alltagsbegleitern, die rund um die Uhr in der Wohnung anwesend sind, entscheiden die Bewohnerinnen und Bewohner selbst, wann sie aufstehen und frühstücken, wie sie den Tag verbringen und wann sie zu Bett gehen möchten. Mitarbeitende des Pflegeteams kommen - wie ein ambulanter Dienst im häuslichen Umfeld - in die Wohnung und unterstützen pflegebedürftige Bewohnerinnen und
Bewohner bei der umfassenden Körperpflege, führen ärztliche Verordnungen aus und kümmern sich um die Medikation. Anschließend verlassen die Pflegemitarbeitenden die Wohnung wieder, sind aber im Bedarfsfall jederzeit erreichbar.

Ambulante Wohngemeinschaften eignen sich auch für schwer pflegebedürftige Personen und Menschen mit Demenz. Bei Vorliegen einer Pflegestufe erhalten die Bewohner Zuschüsse der Pflegekasse auf ambulantem Niveau.

www.wohlfahrtswerk.de 
dar. Folglich ist anzunehmen, dass aus finanziellen Gründen eine DemenzWohngemeinschaft nicht für jeden Menschen eine Alternative zum Altenpflegeheim darstellen kann.

Sofern also Demenz-Wohngemeinschaften in Zukunft keine exklusive, sondern eine breit zugängliche Wohnund Versorgungsform sein sollen, müssen sich auch diese intensiver mit betriebswirtschaftlichen Instrumenten auseinandersetzen. (12)

\section{Ausblick}

Demenz-Wohngemeinschaften stellen schon jetzt nicht mehr nur eine Ergänzung der Versorgung von demenzerkrankten Menschen dar, sondern ansatzweise einen Ersatz für die stationäre Versorgung in Altenpflegeheimen. Aus betriebswirtschaftlicher Sicht werden sich Demenz-Wohngemeinschaften zunehmend mit Effizienzpotenzialen auseinandersetzen müssen. Eine Auswahl möglicher Ansatzpunkte wäre:

- Verbundeffekte von Einkaufsgemeinschaften mit anderen Einrichtungen

- Verbesserung der Prozesse und Abläufe in der Verwaltung, Pflege und Betreuung

- Bildung von Kooperationsmodellen, beispielsweise mit anderen Wohngemeinschaften, als auch mit informellen und ehrenamtlichen Strukturen

- Analyse von Skaleneffekten (Was ist die optimale Zahl an Bewohnern?)

- Energieeffizienz von Gebäuden, Anlagen, Räumlichkeiten und Geräten

Die derzeitigen Forschungsergebnisse lassen durchaus vermuten, dass die Pflege und Betreuung von demenzkranken Menschen in kleinen, familienähnlichen Wohneinheiten mit der ökonomischen Effizienz vereinbar sein könnten, und nicht zwangsläufig zueinander in Widerspruch stehen.

\section{Anmerkungen}

(1) Vgl. Förtsch/Förstl/Gratzl-Pabst 2011, S. 534 ff.

(2) PNG - Gesetz zur Neuausrichtung der Pflegeversicherung (PflegeNeuausrichtungs-Gesetz). Bundesdrucksache 17/10157.

(3) Vgl. Kremer-Preiß/Narten 2004.

(4) Vgl. Wolf-Ostermann 2007.
(5) Vgl. Kremer-Preiß/Narten 2004, S. 23; Wolf-Ostermann 2007, S. 85.

(6) Vgl. Kremer-Preiß/Narten 2004, S. 40; Wolf-Ostermann 2007, S. 84.

(7) Vgl. Wolf-Ostermann 2007, S. 111.

(8) Vgl. ebd.

(9) Vgl. Kremer-Preiß/Narten 2004, S. 67.

(10) Vgl. Statistisches Bundesamt 2011; Augurzky et al. 2008.

(11) SGB XI - Elftes Buch Sozialgesetzbuch $\mathbb{} 36$ und $\mathbb{4} 43$. Der Leistungssatz bei Pflegestufe 3 unterscheidet sich zwischen ambulanter und stationärer Versorgung nicht (=1.550 Euro).

(12) Vgl. am Beispiel der medizinischen Gesundheitsversorgung Fleßa 2007, S. 5-10.

\section{Literatur}

Augurzky, B./Borchert, L./Deppisch, R. et al. (2008). Heimentgelte bei der stationären Pflege in Nordrhein-Westfalen. Ein Bundesländervergleich. Essen.

Fleßa, S. (2007). Gesundheitsökonomik. Eine Einführung in das wirtschaftliche Denken für Mediziner. 2. Auflage, Berlin und andere Orte. Förtsch, B./Förstl, H./Gratzl-Pabst, E. (2011). Sozialpädagogische Hilfen. In: Förstl, H. Demenzen in Theorie und PraxiS. 3., aktualisierte und überarbeitete Auflage. Berlin u.a.O. 2011,

S. 503-540.

Kremer-Preiß, U./Narten, R. (2004).

Betreute Wohngruppen. Struktur des

Angebots und Aspekte der Leistungsqualität. Bertelsmann Stiftung/Kuratorium Deutsche Altershilfe (Hg.), Köln.

PNG - Gesetz zur Neuausrichtung der Pflegeversicherung (Pflege-Neuausrichtungs-Gesetz). Bundesdrucksache 17/10157.

\section{SGB XI Elftes Buch Sozialgesetzbuch - Soziale}

Pflegeversicherung (Artikel 1 des Gesetzes vom 26. Mai 1994, BGBI. I S. 1014), das zuletzt durch Artikel 2C des Gesetzes vom 21. Juli 2012 (BGBI. I S. 1601) geändert worden ist.

Statistisches Bundesamt (Hg.) (2011). Pflegestatistik 2009. Pflege im Rahmen der Pflegeversicherung Deutschlandergebnisse. Wiesbaden

Wolf-Ostermann, K. (2007). Berliner Studie zu Wohngemeinschaften für pflegebedürftige Menschen. Berlin.

\section{Zuverlässiger Begleiter im Adoptionsverfahren}

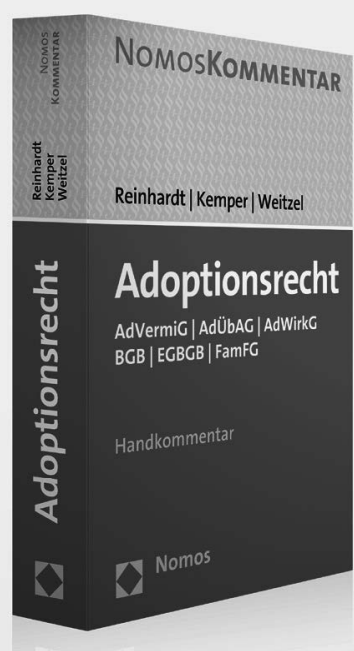

\author{
Adoptionsrecht \\ AdVermiG | AdÜbAG | \\ AdWirkG | BGB | EGBGB | \\ FamFG \\ Handkommentar \\ Von Prof. Jörg Reinhardt, \\ Dr. Rainer Kemper und \\ Wolfgang Weitzel \\ 2012, 270 S., brosch., 39,- $€$ \\ ISBN 978-3-8329-7246-2
}

Der Handkommentar erläutert erstmals im Zusammenhang alle Bestimmungen, die für die Zeit von der Bewerbung über das Vermittlungsverfahren bis zum rechtskräftigen Abschluss der Adoption von Bedeutung sind: AdVermiG, AdÜbAG, BGB, EGBGB, FamFG, AdWirkG. Berücksichtigt wird auch die internationale Adoption, deren Bedeutung angesichts der geringen Zahl der Inlandsadoptionen stetig wächst.

www.nomos-shop.de/14399

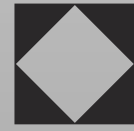

